\title{
Time asymmetric spacetimes near null and spatial infinity. II. Expansions of developments of initial data sets with non-smooth conformal metrics.
}

\author{
Juan Antonio Valiente Kroon * \\ Institut für Theoretische Physik, \\ Universität Wien, \\ Boltzmanngasse 5, A-1090 Wien, \\ Austria.
}

July 25, 2018

\begin{abstract}
This article uses the conformal Einstein equations and the conformal representation of spatial infinity introduced by Friedrich to analyse the behaviour of the gravitational field near null and spatial infinity for the development of initial data which are, in principle, nonconformally flat and time asymmetric. This article is the continuation of the investigation started in Class. Quantum Grav. 21 (2004) 5457-5492, where only conformally flat initial data sets were considered. For the purposes of this investigation, the conformal metric of the initial hypersurface is assumed to have a very particular type of non-smoothness at infinity in order to allow for the presence of non-Schwarzschildean initial data sets in the class under study. The calculation of asymptotic expansions of the development of these initial data sets reveals - as in the conformally flat case - the existence of a hierarchy of obstructions to the smoothness of null infinity which are expressible in terms of the initial data. This allows for the possibility of having spacetimes where future and past null infinity have different degrees of smoothness. A conjecture regarding the general structure of the hierarchy of obstructions is presented.
\end{abstract}

Pacs: 04.20.Ha, 04.20.Ex

\section{Introduction}

This article is the second part of an investigation of the gravitational field near null and spatial infinity arising as the time development of initial data sets which are not time symmetric - that is, the second fundamental form of the initial data set is non-vanishing. Part I of this study was concerned with the analysis of initial data sets for which the 3-metric of the initial hypersurface -its first fundamental form - was assumed to be conformally flat [17. The main result of part I was the uncovering of a time asymmetric hierarchy of obstructions to the smoothness of null infinity, which if vanishing, would imply that the initial data set under consideration is asymptotically Schwarzschildean to a certain order. The obstructions found in part I are the direct generalisations of those found in [15] for time symmetric, conformally flat initial data. In hindsight, the analysis performed in [15] could be readily generalised in two directions: (i) removing the conformal flatness assumption, but keeping the time symmetry; (ii) keeping the conformal flatness of the initial 3-metric, but taking away the time symmetry; (iii) doing without the conformal flatness and time symmetry. The generalisation (i) was carried out in 14, where a

*E-mail address: jav@ap.univie.ac.at 
hierarchy of obstructions implying asymptotic staticity of the initial data near infinity was found. As mentioned earlier, generalisation (ii) was carried out in part I.

This article is essentially concerned with generalisation (iii). That is, we want to deduce asymptotic expansions of the gravitational field near null and spatial infinity for the developments of time asymmetric initial data sets which are not conformally flat. From the results in [15, 14 and part I, we expect that the stationary spacetimes will play a crucial role in our analysis. As we are constructing our asymptotic expansions out of Cauchy initial data sets, we are in the need of considering a class of initial data big enough to contain strictly stationary data - i.e. not only disguised static data. From the results concerning the non-existence of conformally flat initial slices in the Kerr and other stationary solutions, it is clear that with conformally flat data, one would only be dealing with - more or less sophisticated - Schwarzschildean data [13. Thus, one has to move away from conformal flatness. But this is not enough, for in [4 it was shown that the conformal metric of Kerrian initial data is actually non-smooth - a feature that is bound to pervade all stationary solutions with non-vanishing angular momentum.

The general strategy of this article can be summarised as follows: given a sufficiently wide class of initial data sets, we calculate asymptotic expansions for the initial data of the conformal Einstein equations. These expansions are, in turn, fed into the conformal evolution equations and used to obtain in a recursive way asymptotic expansions of the development of the initial data sets. These expansions describe the behaviour of the resulting spacetime in the region close to null and spatial infinity. The recursive nature of the calculations is quite amenable for the implementation on a computer algebra (CA) system. Indeed, the crucial results given in this article have been calculated using scripts written in the system Maple V.

As in part I, the asymptotic expansions show generically a certain type of logarithmic nonsmoothness at the sets where null infinity and spatial infinity "touch". Because of the hyperbolic nature of the evolution part of the conformal field equations it is quite likely that this non-smoothness will be propagated along the generators of null infinity. Hence, the conformal completion will be non-smooth. A remarkable feature of our set up is that it allows us to identify the parts of the initial data responsible for these logarithmic divergences. As a consequence, one obtains a hierarchy of quantities expressible in terms of the initial data - henceforth to be referred to as an obstruction - whose vanishing would eliminate a certain type of logarithmic divergences.The hierarchy exhibits a subtle structure. Actually, the regularity observed in our calculations permits to infer what the general behaviour of the obstructions to any order should be.

In part I, the relative simplicity of the conformal data permitted to show that the vanishing of the obstructions to a certain order leads to an initial data set which is asymptotically Schwarzschildean - but not necessarily time symmetric. In the case of the current article, the increased complexity of the initial data sets considered, and of the resulting expansions preclude us from deducing an analogous condition with regards to stationarity. That is, if one were able to carry the expansions to high enough order one would expect to be able to deduce asymptotic stationarity from the hierarchy of obstructions. An analysis of how stationary data and their asymptotic expansions fit in the framework of the cylinder at spatial infinity will be pursued elsewhere.

As a continuation to [17], this article is completely consistent with the notation, conventions and nomenclature of part I. The main features of our set up, including the construction of the cylinder at infinity and the use of 2-spinors have been discussed there and the reader will be duly referred to it whenever it is necessary. The article is structured as follows: in section 2 , we discuss the existence of solutions to the constraint equations which are expandable in the asymptotic region in terms of powers of $1 / r$. The discussion of this section is based in partially unpublished results due to S.Dain. As we are using systematically a 2 -spinor formalism, in section 3 , we discuss the effects of our class of initial data on the solutions of the structure equations. In section 4 , it is shown how to fix the conformal gauge in the initial data. This is done by resorting to a certain gauge based on conformal geodesics on the initial hypersurface (the cn-gauge). This procedure of fixing the conformal gauge is important if one wants to identify the parts of the freely specifiable data which are pure gauge. Section 5 is concerned with the expansions of solutions of the non-conformally flat momentum constraint. Section 6 does likewise but now with the 
solutions of the Hamiltonian constraint. Section 7 discusses how a certain regularity condition first found by H. Friedrich can be implemented in our set up. Section 8 is concerned with the solutions of the transport equations implied by the conformal Einstein equations on the cylinder at spatial infinity from which our asymptotic expansions are deduced. Section 9 speculates about the general behaviour of the hierarchy of obstructions and the associated logarithmic divergences of the solutions to the transport equations at the sets where null infinity "touches" spatial infinity. Finally, section 10 provides some conclusions.

In order to carry out the calculations here described, a number of assumptions regarding the initial data set have been made. These are clearly numbered from 1 to 5 .

\subsection{General conventions}

Throughout, we shall fully follow the conventions used in part I. In particular, let $\left(\widetilde{\mathcal{M}}, \widetilde{g}_{\mu \nu}\right)$ denote a spacetime arising as the development of some Cauchy initial data $\left(\widetilde{\mathcal{S}}, \widetilde{h}_{\alpha \beta}, \widetilde{\chi}_{\alpha \beta}\right)$. Tilded quantities will refer to quantities in the physical spacetime, whereas untilded ones will denote generically quantities on an unphysical - i.e. conformally rescaled - spacetime. The indices $\mu, \nu$, $\lambda, \ldots$ (second half of the Greek alphabet) are spacetime indices taking the values $0, \ldots, 3$; while $\alpha, \beta, \gamma, \ldots$ are spatial ones with range $1, \ldots, 3$. The latin indices $a, b, c, \ldots$ will be used in spatial expressions which are valid for a particular coordinate system (usually a Cartesian normal one) and take the values $1, \ldots, 3$. The indices, $i, j, k, \ldots$ are spatial frame indices ranging $1, \ldots, 3$, while $A, B, C, \ldots$ will be spinorial indices taking the values 0,1 . Because of the use of spinors, the signature of $\widetilde{g}_{\mu \nu}$ will be taken to be $(+,-,-,-)$, and the 3 -dimensional metric $\widetilde{h}_{\alpha \beta}$ will be negative definite.

We shall be concentrating in a particular asymptotically flat end of the manifold $\mathcal{S}$. Without loss of generality, we shall assume that there is only one asymptotic end. Let $\left\{y^{a}\right\}$ denote coordinates adapted to that end in the sense that $\widetilde{h}_{a b}=-\delta_{a b}+\mathcal{O}(1 /|y|)$ as $|y| \rightarrow \infty$. We shall also assume $\left(\widetilde{\mathcal{S}}, \widetilde{h}_{\alpha \beta}\right)$ to be asymptotically Euclidean and regular in the sense described in part I. Accordingly, let $\left(\mathcal{S}, h_{\alpha \beta}\right)$ denote the 3-dimensional, orientable, smooth compact Riemannian manifold and $i \in \mathcal{S}$ the point whose suitable punctured neighbourhoods correspond to the asymptotically flat end of $\left(\widetilde{\mathcal{S}}, h_{\alpha \beta}\right)$. The manifold $\mathcal{\mathcal { S }}$ is identified in a standard way with $\mathcal{S} \backslash\{i\}$. Most of our discussion shall be carried out in a sufficiently small neighbourhood $\mathcal{B}_{a}(i)$ of radius a centred on $i$. The neighbourhood $\mathcal{B}_{a}(i)$ will be assumed to be geodesically convex. Unless otherwise stated, $\left\{x^{a}\right\}$ denote some normal coordinates with origin at $i$ based on a $h$-orthonormal frame $\left\{e_{j}\right\}$.

A crucial feature of our analysis is that the region of spacetime near null and spatial infinity can be described by means of a certain manifold $\mathcal{M}_{a, \kappa}$ given by

$$
\mathcal{M}_{a, \kappa}=\left\{(\tau, q) \mid q \in \mathcal{C}_{a, \rho},-\frac{\omega(q)}{\kappa(q)} \leq \tau \leq \frac{\omega(q)}{\kappa(q)}\right\}
$$

where $\mathcal{C}_{a, \kappa}=\rho^{1 / 2} \mathcal{C}_{a}$ and $\mathcal{C}_{a}$ is the blow up of the neighbourhood $\mathcal{B}_{a}(i)$. The conformal factor associated with this representation of the "unphysical" spacetime is given by

$$
\Theta=\kappa^{-1} \Omega\left(1-\tau^{2} \frac{\kappa^{2}}{\omega^{2}}\right)
$$

where $\Omega$ is the conformal factor of the initial hypersurface and

$$
\omega=\frac{2 \Omega}{\sqrt{\left|D_{\alpha} \Omega D^{\alpha} \Omega\right|}}
$$

The function $\kappa$ containing the remaining freedom in our setting will be set equal to

$$
\kappa=\rho,
$$

in order to ease our calculations. Accordingly, the critical sets, $\mathcal{I}^{ \pm}$, where null infinity "touches" spatial infinity are given by the point where $\rho=0$ and $\tau= \pm 1$. 
In order to ease the presentation and understanding of the results described in this article, we shall be making the following assumption

Assumption 0 (axial symmetry). The initial data sets $\left(\widetilde{\mathcal{S}}, \widetilde{h}_{\alpha \beta}, \widetilde{\chi}_{\alpha \beta}\right)$ to be considered will be assumed to be axially symmetric.

It follows from well known results - see e.g. [12 — that the development of the initial data will inherit the axial symmetry. It must be stressed that there is no fundamental restriction implied by the latter assumption. All the results presented in this article can be extended naturally - aside from the associated increase of computational complexity - to the non-axially symmetric case.

\section{Existence of solutions to the constraint equations with non-smooth conformal metrics}

As discussed in part I, for technical reasons, we are interested in solutions $\left(\widetilde{h}_{\alpha \beta}, \widetilde{\chi}_{\alpha \beta}\right)$ to the Einstein constraint equations

$$
\begin{aligned}
& \widetilde{r}-\widetilde{\chi}^{2}+\widetilde{\chi}_{\alpha \beta} \widetilde{\chi}^{\alpha \beta}=0, \\
& \widetilde{D}^{\alpha} \widetilde{\chi}_{\alpha \beta}-\widetilde{D}_{\beta} \widetilde{\chi}=0,
\end{aligned}
$$

which on an asymptotic end of the initial hypersurface $\widetilde{\mathcal{S}}$ admit expansions of the form

$$
\widetilde{h}_{\alpha \beta} \sim\left(1+\frac{2 m}{|y|}\right) \delta_{\alpha \beta}+\sum_{k \geq 2} \frac{\widetilde{h}_{\alpha \beta}^{(k)}}{|y|^{k}}, \quad \tilde{\chi}_{\alpha \beta} \sim \sum_{k \geq 2} \frac{\widetilde{\chi}_{\alpha \beta}^{(k)}}{|y|^{k}},
$$

where $\widetilde{h}_{\alpha \beta}^{(k)}$ and $\widetilde{\chi}_{\alpha \beta}^{(k)}$ are smooth functions on the sphere.

We shall perform our analysis not in the physical spacetime which arises as the time development of $\left(\widetilde{\mathcal{S}}, \widetilde{h}_{\alpha \beta}, \widetilde{\chi}_{\alpha \beta}\right)$, but in a conformally rescaled version thereof - the unphysical spacetime. Writing as usual

$$
g_{\mu \nu}=\Omega^{2} \widetilde{g}_{\mu \nu},
$$

we make the following assumption (which was also made in part I):

Assumption 1 (maximal initial data). It shall be assumed that

$$
\Sigma=0, \quad \Omega \chi=\tilde{\chi}=0 \text { on } \widetilde{\mathcal{S}},
$$

where $\Sigma$ denotes the derivative of $\Omega$ along the future $g$-unit normal of $\widetilde{\mathcal{S}}$.

Under the latter assumption, the constraint equations (5a)-(5b) imply on the unphysical manifold $\mathcal{S}$ the equations

$$
\begin{aligned}
& \left(D_{\alpha} D^{\alpha}-\frac{1}{8} r\right) \vartheta=\frac{1}{8} \psi_{\alpha \beta} \psi^{\alpha \beta} \vartheta \text { with } \vartheta=\Omega^{-1 / 2}, \\
& D^{\alpha}\left(\psi_{\alpha \beta}\right)=0,
\end{aligned}
$$

where $D$ is the connection associated with the conformal 3-metric

$$
h_{\alpha \beta}=\Omega^{2} \widetilde{h}_{\alpha \beta},
$$

and the 3 -tensor $\psi_{\alpha \beta}$ is given by

$$
\psi_{\alpha \beta}=\Omega^{-1} \widetilde{\chi}_{\alpha \beta}=\vartheta^{2} \widetilde{\chi}_{\alpha \beta} .
$$

The equations (9a) and (9b) are to be supplemented with the asymptotic (boundary) conditions

$$
|x| \vartheta \rightarrow 1, \quad \psi_{\alpha \beta}=\mathcal{O}\left(\frac{1}{|x|^{4}}\right), \quad \text { as }|x| \rightarrow 0,
$$


where $\left\{x^{a}\right\}$ are normal coordinates with origin at $i$.

Conditions for the existence of solutions to the equations (9a)conditions, and such that the corresponding physical fields have the asymptotic behaviour (6) have been given in [7] under the assumption that the conformal metric $\widetilde{h}_{\alpha \beta}$ is smooth in a neighbourhood $\mathcal{B}_{a}(i)$ of infinity with respect to some normal coordinates. Unfortunately, it seems that the assumption of smoothness of the conformal metric is not enough to discuss stationary spacetimes. In [4] it has been shown that for Kerrian data derived from the Kerr spacetime in Boyer-Lindquist coordinates,

$$
h_{a b}=h_{a b}^{(I)}+|x|^{3} h_{a b}^{(I I)},
$$

where $h_{a b}^{(I)}$ and $h_{a b}^{(I I)}$ are analytic in $\mathcal{B}_{a}(i)$. The important fact to be noted here is that $|x|^{3} \in$ $C^{2, \alpha}\left(\mathcal{B}_{a}(i)\right)$, whence also $h_{a b} \in C^{2, \alpha}\left(\mathcal{B}_{a}(i)\right)$. In the light of the results given in 3 , 13 it is very unlikely that there are slices in the Kerr solution with a better smoothness at infinity. Now, stationary spacetimes are, to leading order, Kerrian [1, and thus, similar non-smoothness is to be expected from any other stationary solution with non-vanishing angular momentum.

Existence results for solutions to the equations (9a) and (9b) under assumption of a conformal metric of the form given in equation (13) and which are expandable in powers of $|x|$ are only available under the premise of axial symmetry [6, 2. Nevertheless, results for the non-axisymmetric case seem plausible.

Dain's existence results use the fact that for axially symmetric initial data sets it is possible to explicitly write solutions to the momentum constraint. These solutions are calculated from freely a specifiable function which acts as a potential. So, if the metric $h_{a b}$ has an axial Killing vector $\eta^{a}$, then a solution of the momentum constraint, equation (9b) is given by

$$
\psi^{a b}=\frac{2 \psi^{(a} \eta^{b)}}{h_{c d} \eta^{c} \eta^{d}}
$$

where

$$
\psi^{a}=\frac{1}{h_{d e} \eta^{d} \eta^{e}} \epsilon^{a b c} \eta_{b} D_{c} \omega, \quad \mathcal{L}_{\eta} \omega=0 .
$$

The potential $\omega$ in the last equation is calculated from a spin 0 real function $\lambda$ and a constant $J^{z}$ via

$$
\omega=\check{\partial}^{2} \lambda \sin ^{2} \theta+J^{z}\left(-3 \cos \theta+\cos ^{3} \theta\right)
$$

where $\partial$ is the standard NP "eth" operator.

It turns out that solutions to the momentum constraint constructed by the aforediscussed procedure satisfy the regularity condition given by Dain \& Friedrich in [7. Indeed,

Theorem 1 (Dain, 2003). Assume that a metric of the form (13) has an axial Killing vector $\eta^{a}$. Let $\psi^{a b}$ be given by [14), (15), and (16). If $|x| \lambda \in E^{\infty}\left(B_{a}(i)\right)$ then $|x|^{8} \psi_{a b} \psi^{a b} \in E^{\infty}\left(B_{a}(i)\right)$.

The purpose of the condition $|x|^{8} \psi_{a b} \psi^{a b} \in E^{\infty}\left(B_{a}(i)\right)$ is to ensure that the solutions to the Hamiltonian constraints are free of $\ln |x|$ terms. This is the content of the following

Theorem 2. Let $h_{a b}$ be a metric that on $\mathcal{B}_{a}(i) \in S$ is of the form [13) with $x^{a} h_{a b}^{(I I)}=0$, which is furthermore smooth on $\widetilde{S}=S \backslash\{i\}$. Let $\psi_{a b}$ be given by equations (14), (15), and (16). Then there exists a solution $\vartheta$ to (9a) which is positive, satisfies the boundary conditions (12), and on $B_{a}(i)$ has the form

$$
\vartheta=\frac{\hat{\vartheta}}{|x|}, \quad \hat{\vartheta} \in E^{\infty}\left(\mathcal{B}_{a}(i)\right), \quad \hat{\vartheta}(i)=1
$$

\section{The structure equations on $\mathcal{S}$}

Let $\tau^{\mu}=\sqrt{2} e_{0}^{\mu}$, where $e_{0}^{\mu}$ is the future $g$-unit normal of $\mathcal{S}$, and let $S U(\mathcal{S})$ be the bundle of spacespinors associated with $\tau^{A A^{\prime}}$, the spinorial counterpart of $\tau^{\mu}$. In part I, a certain submanifold $\mathcal{C}_{a} \subset S U(\mathcal{S})$ given by

$$
\mathcal{C}_{a}=\{\delta(\rho, t) \in S U(\mathcal{S})|| \rho \mid<a, t \in S U(2, \mathbb{C})\}
$$


was defined. The manifold $\mathcal{C}_{a}$ inherits from $S U(\mathcal{S})$ a $\mathfrak{s u}(2, \mathbb{C})$-valued connection form $\check{\omega}^{A}{ }_{B}$ compatible with the metric $h_{\alpha \beta}$ and 1-form $\sigma^{A B}$, the solder form, such that

$$
h \equiv h_{\alpha \beta} d x^{\alpha} \otimes d x^{\beta}=h_{A B C D} \sigma^{A B} \otimes \sigma^{C D},
$$

where

$$
\sigma^{A B}=\sigma_{1}^{A B} d \rho+\sigma_{+}^{A B} \alpha^{+}+\sigma_{-}^{A B} \alpha^{-} .
$$

Let $c_{C D}$ be the vector fields dual to the forms $\sigma^{A B}$, in the sense that

$$
\left\langle\sigma^{A B}, c_{C D}\right\rangle=h_{C D}^{A B}, \quad c_{C D}=c_{C D}^{1} \partial_{\rho}+c_{C D}^{+} X_{+}+c_{C D}^{-} X_{-},
$$

where

$$
c_{A B}^{1}=x_{A B}, \quad c_{A B}^{+}=\frac{1}{\rho} z_{A B}+\check{c}_{A B}^{+}, \quad c_{A B}^{-}=\frac{1}{\rho} y_{A B}+\check{c}_{A B}^{-} .
$$

General considerations similar to those in [8] require that,

$$
\check{c}_{A B}^{ \pm}=Y^{ \pm} y_{A B}+Z^{ \pm} z_{A B},
$$

The relation (19) shows that a knowledge of the conformal metric $h_{\alpha \beta}$ is equivalent to a knowledge of the solder form $\sigma^{A B}$, and this, in turn, is equivalent to a knowledge of the frame vector field $c_{A B}$. Hence, and because of the systematic use of space spinors in this work, we shall regard the regular parts, $\check{c}_{A B}^{ \pm}$, of the frame coefficients, $c_{A B}^{ \pm}$, as encoding the non-conformal flatness of the conformal metric $h_{\alpha \beta}$.

Now, assuming that the conformal 3-metric, $h_{a b}$ is of the form (13), it follows from the relations (19)-(22) and a "bookkeeping" argument that

$$
\check{c}_{A B}^{ \pm}=\left(\check{c}^{ \pm}\right)_{A B}^{(I)}+\rho\left(\check{c}^{ \pm}\right)_{A B}^{(I I)},
$$

where $\left(\check{c}^{ \pm}\right)_{A B}^{(I)}=\mathcal{O}\left(\rho^{2}\right)$ and $\left(\check{c}^{ \pm}\right)_{A B}^{(I I)}=\mathcal{O}(\rho)$ are analytical spinorial fields on $\mathcal{C}_{a}$. More precisely, in order to have a class of initial data sets large enough to contain stationary data we make the following assumption:

Assumption 2. The frame fields $c_{A B}$ on $\mathcal{C}_{a}$ are such that

$$
\check{c}_{A B}^{ \pm}=\left(\check{c}^{ \pm}\right)_{A B}^{(I)}+\rho\left(\check{c}^{ \pm}\right)_{A B}^{(I I)}
$$

where $\left(\check{c}^{ \pm}\right)_{A B}^{(I)}=\mathcal{O}\left(\rho^{2}\right)$ and $\left(\check{c}^{ \pm}\right)_{A B}^{(I I)}=\mathcal{O}(\rho)$ are analytical spinorial fields on $\mathcal{C}_{a}$.

Remarks. The spin weights of the diverse components of $c_{A B}^{ \pm}$imply under assumption 2 the following expansion Ansätze:

$$
\begin{aligned}
& Y^{+}=\sum_{p=2}^{\infty} \sum_{q=2}^{p} \sum_{m=0}^{2 q} Y_{p ; 2 q, m}^{+} T_{2 q}{ }^{m}{ }_{q+2} \rho^{p}, \quad Y^{-}=\sum_{p=2}^{\infty} \sum_{q=0}^{p} \sum_{m=0}^{2 q} Y_{p ; 2 q, m}^{-} T_{2 q}{ }^{m}{ }_{q} \rho^{p}, \\
& Z^{+}=\sum_{p=2}^{\infty} \sum_{q=2}^{p} \sum_{m=0}^{2 q} Z_{p ; 2 q, m}^{+} T_{2 q}{ }^{m}{ }_{q-2} \rho^{p}, \quad Z^{-}=\sum_{p=2}^{\infty} \sum_{q=0}^{p} \sum_{m=0}^{2 q} Z_{p ; 2 q, m}^{-} T_{2 q}{ }^{m}{ }_{q} \rho^{p},
\end{aligned}
$$

where the coefficients $Y_{p ; 2 q, m}^{ \pm}$and $Z_{p ; 2 q, m}^{ \pm}$are complex numbers satisfying the reality conditions

$$
Z_{p ; 2 q, m}^{-}=(-1)^{m+q} \bar{Y}_{p ; 2 q, 2 q-m}^{+}, \quad Y_{p ; 2 q, m}^{-}=(-1)^{m+q} \bar{Z}_{p ; 2 q, 2 q-m}^{+} .
$$

Note that, for example

$$
\begin{gathered}
Y^{-}=\left(\frac{1}{2 !}\left[Y_{2 ; 0,0}^{-} T_{0}{ }_{0}^{0}+\sum_{m=0}^{4} Y_{2 ; 4, m}^{-} T_{4}{ }_{2}^{m}\right] \rho^{2}+\frac{1}{3 !}\left[\sum_{m=0}^{2} Y_{3 ; 2, m}^{-} T_{2}{ }_{1}^{m}+\sum_{m=0}^{6} Y_{3 ; 6, m}^{-} T_{6}{ }_{3}{ }_{3}\right] \rho^{3}+\cdots\right) \\
+\rho\left(\left[\sum_{m=0}^{2} Y_{2 ; 2, m}^{-} T_{2}{ }^{m} 1\right] \rho+\left[Y_{3 ; 0,0}^{-} T_{0}{ }_{0}^{0}+\sum_{m=0}^{4} Y_{3,4, m}^{-} T_{4}{ }^{m}{ }_{2}\right] \rho^{2}+\cdots\right),
\end{gathered}
$$


so that the analytical function contained within the second pair of round brackets is responsible for the non-analyticity of the frame vectors - and of that of the conformal metric. Similar arguments can be made with the remaining coefficients $Y^{+}, Z^{+}$and $Z^{-}$. More precisely, one has the following

Lemma 1. If the frame fields satisfy assumption 2 then in $\mathcal{B}_{a}(i)$ the conformal metric is of the form

$$
h_{a b}=h_{a b}^{(I)}+|x| h_{a b}^{(I I)},
$$

with $h_{a b}^{(I)}$ and $h_{a b}^{(I I)}$ analytic.

Thus, assumption 2 implies a conformal metric which is rougher at infinity than that of the Kerr data discussed by S. Dain in 4 .

Finally, it is noted that under the assumption of axial symmetry (assumption 0) the Ansätze (24a)-24b) simplify to

$$
\begin{aligned}
& Y^{+}=\sum_{p=2}^{\infty} \sum_{q=2}^{p} Y_{p ; 2 q, q}^{+} T_{2 q}{ }_{q+2}^{q} \rho^{p}, \quad Y^{-}=\sum_{p=2}^{\infty} \sum_{q=0}^{p} Y_{p ; 2 q, q}^{-} T_{2 q}{ }_{q}^{q} \rho^{p}, \\
& Z^{+}=\sum_{p=2}^{\infty} \sum_{q=2}^{p} Z_{p ; 2 q, q}^{+} T_{2 q q}^{q}{ }^{q} \rho^{p}, \quad Z^{-}=\sum_{p=2}^{\infty} \sum_{q=0}^{p} Z_{p ; 2 q, q}^{-} T_{2 q q}{ }^{q} \rho^{p},
\end{aligned}
$$

while the reality condition (25) becomes

$$
Z_{p ; 2 q, q}^{-}=\bar{Y}_{p ; 2 q, q}^{+}, \quad Y_{p ; 2 q, q}^{-}=\bar{Z}_{p ; 2 q, q}^{+} .
$$

\subsection{Expansions for the connection coefficients}

Now, recall that the contraction of the connection form $\check{\omega}^{A}{ }_{B}$ with the frame $c_{A B}$ defines the connection coefficients $\gamma_{C D A B}$. More precisely, we write

$$
\gamma_{C D A B} \equiv\left\langle\check{\omega}_{B}^{E}, c_{C D}\right\rangle \epsilon_{E A}=\frac{1}{2 \rho}\left(\epsilon_{A C} x_{B D}+\epsilon_{B D} x_{A C}\right)+\check{\gamma}_{C D A B}=\frac{1}{\rho} \gamma_{C D A B}^{*}+\check{\gamma}_{C D A B},
$$

where

$$
\check{\gamma}_{01 C D}=0 \text {. }
$$

Moreover, it can be checked that

$$
\check{\gamma}_{1100}=-\check{\gamma}_{0011}, \quad \check{\gamma}_{0010}=\check{\gamma}_{0001}, \quad \check{\gamma}_{1110}=\check{\gamma}_{1101} .
$$

As seen in part I, the regular part of the connection coefficients, $\check{\gamma}_{C D}{ }_{B}{ }_{B}$ vanishes if the conformal metric is flat. However, if the conformal metric satsifies assumption 2 then the connection coefficients depend on the frame coefficients $\check{c}_{A B}^{ \pm}$. In order to calculate the connection coefficients we make use of the commutator equations

$$
\left[c_{A B}, c_{C D}\right]=2\left(\gamma_{A B}{ }^{E}{ }_{(C} \epsilon_{D)}^{F}-\gamma_{C D}{ }^{E}{ }_{(A} \epsilon_{B)}^{F}\right) c_{E F}+\left(c_{A B}^{-} c_{C D}^{+}-c_{C D}^{-} c_{A B}^{+}\right) X .
$$

The latter imply the radial equations,

$$
\begin{aligned}
& \frac{1}{\sqrt{2}} \partial_{\rho}\left(\rho \check{c}_{A A}^{+}\right)=\check{\gamma}_{A A 00}\left(\rho \check{c}_{11}^{+}\right)-\check{\gamma}_{A A 11}\left(\rho \check{c}_{00}^{+}\right)-\frac{1}{\sqrt{2}} \check{\gamma}_{A A 11}, \\
& \frac{1}{\sqrt{2}} \partial_{\rho}\left(\rho \check{c}_{A A}^{-}\right)=\check{\gamma}_{A A 00}\left(\rho \check{c}_{11}^{-}\right)-\check{\gamma}_{A A 11}\left(\rho \check{c}_{00}^{-}\right)-\frac{1}{\sqrt{2}} \check{\gamma}_{A A 00}
\end{aligned}
$$

The latter equations can be used to obtain expansions for the connection coefficients $\check{\gamma}_{0000}$, $\check{\gamma}_{0011}=-\check{\gamma}_{1100}$ and $\check{\gamma}_{1111}$ in terms of the coefficients in the expansions of $Y^{+}, Y^{-}$, and $Z^{-}$. They also show that the frame component $Z^{+}$is not independent, but can also be expressed in terms of $Y^{+}, Y^{-}$, and $Z^{-}$. Furthermore, one has the following 
Lemma 2. Under assumption 2 it follows that the connection coefficents $\gamma_{0000}, \gamma_{0011}, \gamma_{1100}$ and $\gamma_{1111}$ are such that

$$
\check{\gamma}_{A B C D}=\left(\check{\gamma}_{A B C D}\right)^{(I)}+\rho\left(\check{\gamma}_{A B C D}\right)^{(I I)},
$$

where $\left(\check{\gamma}_{A B C D}\right)^{(I)}=\mathcal{O}\left(\rho^{2}\right)$ and $\left(\check{\gamma}_{A B C D}\right)^{(I I)}=\mathcal{O}(\rho)$ are analytic fields on $\mathcal{C}_{a}$. Furthermore, the reality condition $\left(\gamma_{A B C D}\right)^{+}=-\gamma_{A B C D}$ is automatically satisfied if the conditions 25 hold.

The proof of this lemma follows again from a "bookkeeping" argument. Note that the connection coefficients $\gamma_{0001}$ and $\gamma_{1110}$ are not fixed by the procedure we have discussed above. We shall come back to them later.

\subsection{The curvature spinors}

In order to determine expansions for diverse components of the curvature we shall make use of the structure equations. Evaluating the second structure equation - equation (36b) in part I on $c_{C D} \wedge c_{E F}$ one obtains the following radial equations

$$
\begin{aligned}
\frac{1}{\sqrt{2}} \partial_{\rho} \check{\gamma}_{00 A B}+\frac{1}{\rho} & \left\{\check{\gamma}_{0000} z_{A B}-\check{\gamma}_{0011} y_{A B}+\frac{1}{\sqrt{2}} \check{\gamma}_{00 A B}\right\} \\
& =\check{\gamma}_{0000} \check{\gamma}_{11 A B}-\check{\gamma}_{0011} \check{\gamma}_{00 A B}-\frac{1}{2} s_{A B 00}-\frac{1}{6 \sqrt{2}} r y_{A B}, \\
\frac{1}{\sqrt{2}} \partial_{\rho} \check{\gamma}_{11 A B}+\frac{1}{\rho} & \left\{\check{\gamma}_{1100} z_{A B}-\check{\gamma}_{1111} y_{A B}+\frac{1}{\sqrt{2}} \check{\gamma}_{11 A B}\right\} \\
& =\check{\gamma}_{1100} \check{\gamma}_{11 A B}-\check{\gamma}_{1111} \check{\gamma}_{00 A B}-\frac{1}{2} s_{A B 11}-\frac{1}{6 \sqrt{2}} r z_{A B},
\end{aligned}
$$

where $s_{A B C D}$ denotes the spinorial representation of the tracefree part of the Ricci tensor of $h_{\alpha \beta}$, that is,

$$
s_{A B C D}=\sigma_{A B}^{i} \sigma_{C D}^{j} e_{i}^{\alpha} e_{j}^{\beta}\left(r_{\alpha \beta}-\frac{1}{3} r h_{\alpha \beta}\right),
$$

where $r_{\alpha \beta}$ is the Ricci tensor of $h_{\alpha \beta}$ and $r=h^{\alpha \beta} r_{\alpha \beta}$. Because of its symmetries, one can write

$$
s_{A B C D}=s_{0} \epsilon_{A B C D}^{0}+s_{1} \epsilon_{A B C D}^{1}+s_{2} \epsilon_{A B C D}^{2}+s_{3} \epsilon_{A B C D}^{3}+s_{4} \epsilon_{A B C D}^{4} .
$$

The equations (34a) and (34b) have been used to obtain expansions for the components of $s_{A B C D}$ in terms of $Y^{+}, Y^{-}$, and $Z^{-}$. More precisely, one has the following

Lemma 3. Under assumption 0 one has that the components of the Ricci spinor are of the form

$$
s_{j}=s_{j}^{(I)}+\frac{1}{\rho} s_{j}^{(I I)}
$$

where $s_{j}^{(I)}=\mathcal{O}(\rho)$ and $s_{j}^{(I I)}=\mathcal{O}\left(\rho^{2}\right)$ are analytic fields on $\mathcal{C}_{a}$. The reality condition $\left(s_{A B C D}\right)^{+}=$ $s_{A B C D}$ is satisfied if 25 holds.

The tracefree part of the Ricci tensor and the Ricci curvature scalar are not independent from each other, but related via the Bianchi identity

$$
D^{A B} s_{A B C D}=\frac{1}{6} D_{C D} r .
$$

The latter identity implies three equations which can be used to write the Ricci scalar $r$ and the connection coefficients $\gamma_{0001}$ and $\gamma_{1110}$ in terms of the frame freely specifiable data -i.e. $Y^{+}$, $Y^{-}$, and $Z^{-}$. It follows that 
Lemma 4. Under assumption 2 it follows that the Ricci scalar is of the form

$$
r=r^{(I)}+\frac{1}{\rho} r^{(I I)}
$$

where $r^{(I)}=\mathcal{O}(\rho)$ and $r^{(I I)}=\mathcal{O}\left(\rho^{2}\right)$ are analytic fields on $\mathcal{C}_{a}$. Furthermore, the components $\gamma_{0001}$ and $\gamma_{1110}$ of the connection are such that

$$
\check{\gamma}_{A B C D}=\left(\check{\gamma}_{A B C D}\right)^{(I)}+\rho\left(\check{\gamma}_{A B C D}\right)^{(I I)},
$$

where $\left(\check{\gamma}_{A B C D}\right)^{(I)}=\mathcal{O}\left(\rho^{2}\right)$ and $\left(\check{\gamma}_{A B C D}\right)^{(I I)}=\mathcal{O}(\rho)$ are analytic fields on $\mathcal{C}_{a}$. Furthermore, the reality condition $\left(\gamma_{A B C D}\right)^{+}=-\gamma_{A B C D}$ is automatically satisfied if the conditions 25 hold.

\section{Fixing the conformal gauge on $\mathcal{S}$}

In our set up, the functions $Y^{+}, Y^{-}$, and $Z^{-}$determining the frame - via the relations (21) and (22) - are used to specify the part of the free data which is more commonly encoded in the metric. In their expansions, these functions contain terms which are pure gauge. The reason for this is the following: given $\vartheta$ and $\psi_{\alpha \beta}$ solutions of the constraint equations (9a) and (9b) and a positive function, $\phi$, on $\mathcal{S}$, it is well known that the transition

$$
h_{\alpha \beta} \mapsto \phi^{4} h_{\alpha \beta}, \quad \psi_{\alpha \beta} \mapsto \phi^{-2} \psi_{\alpha \beta}, \quad \vartheta \mapsto \phi^{-1} \vartheta, \quad \chi_{\alpha \beta} \mapsto \phi^{2} \chi_{\alpha \beta}
$$

yields another solution to the Einstein constraint equations with the same physical data. In order to fix this conformal freedom we shall use a certain gauge based on spacelike conformal geodesics starting at $i$. We shall refer to this gauge as the cn-gauge.

The cn-gauge is defined as follows: consider the 3-dimensional conformal geodesic equations,

$$
\begin{aligned}
& \dot{x}^{\beta} \nabla_{\beta} \dot{x}^{\alpha}=-2 b_{\beta} \dot{x}^{\beta} \dot{x}^{\alpha}+\dot{x}_{\beta} \dot{x}^{\beta} b^{\alpha}, \\
& \dot{x}^{\beta} \nabla_{\beta} b_{\alpha}=b_{\beta} \dot{x}^{\beta} b_{\alpha}-\frac{1}{2} b_{\beta} b^{\beta} \dot{x}_{\alpha}+\left(s_{\alpha \beta}+\frac{1}{12} r h_{\alpha \beta}\right) \dot{x}^{\beta},
\end{aligned}
$$

where $x^{\alpha}(t)$ is a curve on $\mathcal{S}$, and $b^{\alpha}$ an associated 3 -dimensional 1 -form. We supplement the conformal equations with the initial conditions,

$$
x(0)=i, \quad \dot{x}_{\beta} \dot{x}^{\beta}=-1, \quad b(0)=0 .
$$

If $a$ is chosen small enough, there exists a unique solution to these equations on $\mathcal{B}_{a}(i)$. Furthermore, there exists in $\mathcal{B}_{a}(i)$ an unique conformal rescaling such that,

$$
b_{\beta} \dot{x}^{\beta}=0 \quad \text { on } \mathcal{B}_{a}(i),
$$

can always be found.

Definition. A metric in the conformal class for which the condition 40 is satisfied along the solutions of the 3-dimensional conformal geodesic equations (37) and (38) will be said to be in the cn-gauge.

It is noticed that for conformally flat data, if $\dot{x}$ is the tangent to (standard) geodesics starting at $i$ with $\dot{x}_{\beta} \dot{x}^{\beta}=-1$, and if one requires $b \equiv 0$ in $\mathcal{B}_{a}(i)$, one is automatically in the cn-gauge.

Let $\dot{X}_{A B}$ and $B_{A B}$ be, respectively, the space spinors corresponding to the 3-dimensional vector $\dot{x}^{\alpha}$ and the 1 -form $b_{\alpha}$. These can be written as,

$$
\dot{X}_{A B}=\dot{X}_{x} x_{A B}+\dot{X}_{y} y_{A B}+\dot{X}_{z} z_{A B}, \quad B_{A B}=B_{x} x_{A B}+B_{y} y_{A B}+B_{z} z_{A B} .
$$

Using these two spinors and the spatial Infeld symbols, one can produce a spinorial version of the conformal geodesic equations (37) and (38). 
Following our overall strategy, one can attempt tosolve the 3-dimensional conformal geodesic equations together with the cn-gauge assumption by means of formal expansions. Spin weight considerations plus the initial data for the conformal geodesics show that

$$
\dot{X}_{x}=1+\mathcal{O}(\rho), \quad \dot{X}_{y}=\mathcal{O}(\rho), \quad \dot{X}_{z}=\mathcal{O}(\rho) .
$$

Similarly, for the components of the spinor $B_{A B}$ one has that

$$
B_{x}=\mathcal{O}(\rho), \quad B_{y}=\mathcal{O}(\rho), \quad B_{z}=\mathcal{O}(\rho) .
$$

Once the conformal geodesic equations have been solved, requiring the cn-gauge condition $\dot{x}^{\alpha} b_{\alpha}=$ $\epsilon^{A C} \epsilon^{B D} \dot{X}_{C D} B_{A B}=0$ renders further relations between the coefficients in the expansions of the frame components. Indeed, an explicit calculation with Maple $\mathrm{V}$ shows that

Lemma 5. Under assumption 圆 the cn-gauges imply that the coefficients $Y_{p ; 2 q, m}^{-}$with $2 \leq p \leq 7$, $0 \leq q \leq p, 0 \leq m \leq 2 q$ are determined by the algebraic combinations of the coefficients $Y_{p ; 2 q, m}^{+}$ and $Z_{p ; 2 q, m}^{-}$with $2 \leq p \leq 7,0 \leq q \leq p, 0 \leq m \leq 2 q$ of $Y^{+}$and $Z^{-}$. The only exceptions are the coefficients $Y_{3 ; 2, m}^{-}, m=0,1,2$ and $Y_{3 ; 0,0}^{-}$which remain freely specifiable.

The full details, being not very illuminating, shall not be given here. Further consequences of the cn-gauge are that in the decompositions

$$
r=r^{(I)}+\frac{1}{\rho} r^{(I I)}, \quad s_{j}=s_{j}^{(I)}+\frac{1}{\rho} s_{j}^{(I I)},
$$

with $j=0, \ldots, 4$ the "smooth parts" are of the form

$$
r^{(I)}=\mathcal{O}\left(\rho^{2}\right), \quad s_{j}^{(I)}=\mathcal{O}\left(\rho^{2}\right),
$$

instead of being $\mathcal{O}(\rho)$ — cfr. with lemma 4.7 in [8]. The "non-smooth parts" do not change order. It may also be of interest to note that if the cn-gauge condition holds then

$$
\dot{X}_{x}=\mathcal{O}\left(\rho^{3}\right), \quad \dot{X}_{y}=\mathcal{O}\left(\rho^{3}\right), \quad \dot{X}_{z}=\mathcal{O}\left(\rho^{3}\right),
$$

and

$$
B_{x}=\mathcal{O}\left(\rho^{5}\right), \quad B_{y}=\mathcal{O}\left(\rho^{2}\right), \quad B_{z}=\mathcal{O}\left(\rho^{2}\right) .
$$

\section{Expansions of the momentum constraint}

We are now faced with the problem of choosing a class of solutions of the momentum constraint, equation (9b), which is large enough to encompass the second fundamental forms of stationary solutions with non-vanishing ADM angular momentum. In agreement with the existence results given in section 2 we shall further require that the solutions satisfy the regularity condition $\psi_{\alpha \beta} \psi^{\alpha \beta} \in E^{\infty}\left(\mathcal{B}_{a}(i)\right)$. The latter requirement is essentially satisfied if one considers solutions to the momentum constraint without linear momentum. Our selected class of solutions will be, in turn, lifted to the manifold $\mathcal{C}_{a}$, where formal expansions in terms of powers of $\rho$ will be calculated.

The results by S. Dain discussed in section 2 strongly use the fact that the initial data possesses axial symmetry to explicitly calculate — via a "potential", $\lambda$ - solutions to the momentum constraint (9b). However, with future applications in mind, we are interested in a procedure that can be easily generalised to a non-axially symmetric setting.

For the time being let us write

$$
\psi_{\alpha \beta}=\psi_{\alpha \beta}^{\prime}+\widehat{\psi}_{\alpha \beta}
$$

where both $\psi_{\alpha \beta}^{\prime}$ and $\widehat{\psi}_{\alpha \beta}$ are symmetric, tracefree tensors. The former is a "seed" tensor and the latter an unknown to be determined upon substitution in the momentum constraint. The standard strategy at this point is to write $\widehat{\psi}_{\alpha \beta}$ in terms of the Killing operator of a 1 -form, $v_{\alpha}$ 
- the York splitting. For reasons to be explained in section 7, we will not proceed in this way, but will solve directly for $\psi_{\alpha \beta}$ - see, however, the remarks in subsection 5.1

In order to make contact with the conformally flat case studied in part I, we shall consider as our "seed" tracefree tensor $\psi_{\alpha \beta}^{\prime}$ the solution to the conformally flat momentum constraint used in part I - see assumption 3 in [17. As in part I, let

$$
\begin{aligned}
& \psi_{2}^{A}=-\frac{A}{\rho^{3}} T_{0}{ }_{0}^{0}, \\
& \psi_{0}^{A}=\psi_{1}^{A}=\psi_{3}^{A}=\psi_{4}^{A}=0, \\
& \psi_{1}^{Q}=\frac{3}{\rho^{2}}\left(Q_{2}+i Q_{1}\right) T_{2}{ }_{0}^{0}-\frac{3 \sqrt{2}}{\rho^{2}} Q_{3} T_{2}{ }_{0}^{1}-\frac{3}{\rho^{2}}\left(Q_{2}-i Q_{1}\right) T_{2}{ }^{2}{ }_{0}, \\
& \psi_{2}^{Q}=\frac{9 \sqrt{2}}{2 \rho^{2}}\left(Q_{2}+i Q_{1}\right) T_{2}{ }_{1}^{0}-\frac{9}{\rho^{2}} Q_{3} T_{2}{ }_{1} 1-\frac{9 \sqrt{2}}{2 \rho^{2}}\left(Q_{2}-i Q_{1}\right) T_{2}{ }_{1} \\
& \psi_{3}^{Q}=-\frac{3}{\rho^{2}}\left(Q_{2}-i Q_{1}\right) T_{2}{ }_{2}{ }_{2}-\frac{3 \sqrt{2}}{\rho^{2}} Q_{3} T_{2}{ }_{2}+\frac{3}{\rho^{2}}\left(Q_{2}+i Q_{1}\right) T_{2}{ }_{2}{ }_{2}, \\
& \psi_{0}^{Q}=\psi_{4}^{Q}=0 \text {, } \\
& \psi_{1}^{J}=\frac{6}{\rho^{3}}\left(-J_{1}+i J_{2}\right) T_{2}{ }_{0}^{0}+\frac{6 \sqrt{2}}{\rho^{3}} i J_{3} T_{2}{ }_{0}^{1}-\frac{6}{\rho^{3}}\left(J_{1}+i J_{2}\right) T_{2}{ }_{0}^{2}, \\
& \psi_{2}^{J}=0 \\
& \psi_{3}^{J}=\frac{6}{\rho^{3}}\left(J_{1}+i J_{2}\right) T_{2}{ }_{2}^{2}-\frac{6 \sqrt{2}}{\rho^{3}} i J_{3} T_{2}{ }_{2}{ }^{1}-\frac{6}{\rho^{3}}\left(-J_{1}+i J_{2}\right) T_{2}{ }_{2}^{0}, \\
& \psi_{0}^{J}=\psi_{4}^{J}=0,
\end{aligned}
$$

were $A, J_{1}, J_{2}, J_{3}, Q_{1}, Q_{2}, Q_{3} \in \mathbb{R}$. Recall that assumption 0 -axial symmetry- requires $Q_{1}=Q_{2}=J_{1}=J_{2}=0$. Furthermore, we write

$$
\psi_{j}^{\lambda}=\frac{1}{\rho^{4}} \sum_{n=2}^{\infty} \sum_{q=2}^{n} L_{j, n-4 ; 2 q, q} T_{2 q q-2+j}^{q} \rho^{n}, \quad j=0, \ldots, 4
$$

where

$$
\begin{aligned}
& L_{1, n-4 ; 2 q, q}=\frac{\left(\left(L_{4, n-4 ; 2 q, q}-L_{0, n-4 ; 2 q, q}\right)(q+1) q+4 L_{0, n-4 ; 2 q, q}(n+3)^{2}\right)}{(n+3)(2(n+4)(n+2)-(q+2)(q-1))} \sqrt{(q+2)(q-1)}, \\
& L_{2, n-4 ; 2 q, q}=\frac{3\left(L_{0, n-4 ; 2 q, q}+L_{4, n-4 ; 2 q, q}\right)}{2(n+3)^{2}-q(q+1)} \sqrt{(q+2)(q+1) q(q-1)}, \\
& L_{3, n-4 ; 2 q, q}=\frac{\left(\left(L_{0, n-4 ; 2 q, q}-L_{4, n-4 ; 2 q, q}\right)(q+1) q+4 L_{4, n-4 ; 2 q, q}(n+3)^{2}\right)}{(n+3)(2(n+4)(n+2)-(q+2)(q-1))} \sqrt{(q+2)(q-1)},
\end{aligned}
$$

and $n=2,3,4, \ldots$, axial symmetry (assumption 0 ) already being assumed here. The coefficents $L_{0 ; 2 q, q}$ and $L_{4 ; 2 q, q}$ are arbitrary complex numbers satisfying the reality condition

$$
L_{0 ; 2 q, q}=\bar{L}_{4 ; 2 q, q} .
$$

We shall make the following

Assumption 3. The totally symmetric spinor $\psi_{A B C D}^{\prime}$ associated with the "seed" tracefree tensor $\psi_{\alpha \beta}^{\prime}$ in the York splitting (62) is assumed to be of the form

$$
\psi_{A B C D}^{\prime}=\psi_{A B C D}^{A}+\psi_{A B C D}^{Q}+\psi_{A B C D}^{J}+\psi_{A B C D}^{\lambda},
$$

where

$$
\psi_{A B C D}^{i}=\psi_{0}^{i} \epsilon_{A B C D}^{0}+\psi_{1}^{i} \epsilon_{A B C D}^{1}+\psi_{2}^{i} \epsilon_{A B C D}^{2}+\psi_{3}^{i} \epsilon_{A B C D}^{3}+\psi_{4}^{i} \epsilon_{A B C D}^{4},
$$

and $i$ denotes any of the labels $A, Q, J, \lambda$. 
The symmetric, tracefree tensor $\widehat{\psi}_{\alpha \beta}$ has a spinorial counterpart $\widehat{\psi}_{A B C D}$ of the form

$$
\widehat{\psi}_{A B C D}=\widehat{\psi}_{0}^{i} \epsilon_{A B C D}^{0}+\widehat{\psi}_{1}^{i} \epsilon_{A B C D}^{1}+\widehat{\psi}_{2}^{i} \epsilon_{A B C D}^{2}+\widehat{\psi}_{3}^{i} \epsilon_{A B C D}^{3}+\widehat{\psi}_{4}^{i} \epsilon_{A B C D}^{4}
$$

Under assumption 3 one has that

$$
D^{A B} \psi_{A B C D}^{\prime}=\mathcal{O}(\rho) \text {. }
$$

Consistent with our general strategy of finding solutions to the constraint equations which are expandable in powers of $\rho$ near infinity, we put forward the Ansatz

$$
\widehat{\psi}_{j}=\sum_{p=2}^{\infty} \sum_{q=|2-l|}^{p} \sum_{m=0}^{2 q} \widehat{\psi}_{j, p ; 2 q, m} T_{2 q}^{m}{ }_{q-2+j} \rho^{p},
$$

where the coefficients $\widehat{\psi}_{j, p ; 2 q, m}$ satisfy the required reality conditions - see part I. Under assumption 0 - axial symmetry - our Ansatz simplifies to

$$
\widehat{\psi}_{j}=\sum_{p=2}^{\infty} \sum_{q=|2-l|}^{p} \widehat{\psi}_{j, p ; 2 q, q} T_{2 q q-2+j}^{q} \rho^{p} .
$$

In this case, substitution into the momentum constraint yields algebraic equations for the coefficients $\widehat{\psi}_{j, p ; 2 q, q}$. These allow us to write the coefficients $\widehat{\psi}_{1, p ; 2 q, q}, \widehat{\psi}_{2, p ; 2 q, q}$ and $\widehat{\psi}_{3, p ; 2 q, q}$ in terms of the freely specifiable data and in terms of the coefficients $\psi_{0, p ; 2 q, q}$ and $\psi_{4, p ; 2 q, q}$. The latter are related to each other by the reality condition

$$
\widehat{\psi}_{4, p ; 2 q, q}=\overline{\widehat{\psi}}_{0, p ; 2 q, q} .
$$

The relations discussed in the above paragraph have been explicitly calculated in the CA system Maple V up to order $p=4$ in the Ansatz (60).

\subsection{A remark concerning the York splitting}

For the sake of completeness, it is of interest to analyse what happens if instead of using the procedure described in the previous subsection, one fully uses the York splitting. Accordingly, let us write

$$
\psi_{\alpha \beta}=\psi_{\alpha \beta}^{\prime}+(\mathscr{L} v)_{\alpha \beta},
$$

where

$$
(\mathscr{L} v)_{\alpha \beta}=D_{\alpha} v_{\beta}+D_{\beta} v_{\alpha}-\frac{2}{3} h_{\alpha \beta} D_{\gamma} v^{\gamma}
$$

is the conformal Killing operator. Let $v_{A B}$ be the spinorial counterpart of the 1-form $v_{\alpha}$ on which the conformal Killing operator in the York splitting - equation (62) acts, and write

$$
v_{A B}=v_{x} x_{A B}+v_{y} y_{A B}+v_{z} z_{A B},
$$

where the coefficients $v_{x}, v_{y}$ and $v_{z}$ satisfy the reality conditions

$$
v_{x}=\bar{v}_{x}, \quad v_{y}=\bar{v}_{z} .
$$

Under assumption 3 one has that

$$
D^{A B} \psi_{A B C D}^{\prime}=\mathcal{O}(\rho) .
$$

The latter suggests the following Ansatz for the components of $v_{A B}$ :

$$
\begin{aligned}
v_{x} & =\sum_{p=1}^{\infty} \sum_{q=0}^{p} \sum_{m=0}^{2 q} v_{x, p ; 2 q, m} T_{2 q}{ }^{m} \rho^{p}, \\
v_{y} & =\sum_{p=1}^{\infty} \sum_{q=1}^{p} \sum_{m=0}^{2 q} v_{y, p ; 2 q, m} T_{2 q}{ }^{m}{ }_{q+1} \rho^{p}, \\
v_{z} & =\sum_{p=1}^{\infty} \sum_{q=1}^{p} \sum_{m=0}^{2 q} v_{z, p ; 2 q, m} T_{2 q}{ }^{m}{ }_{q-1} \rho^{p},
\end{aligned}
$$


where because of the reality conditions one has

$$
v_{y, p ; 2 q, m}=(-1)^{q+m+1} \bar{v}_{z, p ; 2 q, m} .
$$

Under assumption 0 - axial symmetry — the above expressions reduce to

$$
\begin{aligned}
& v_{x}=\sum_{p=1}^{\infty} \sum_{q=0}^{p} v_{x, p ; 2 q, q} T_{2 q}{ }_{q}^{q} \rho^{p}, \\
& v_{y}=\sum_{p=1}^{\infty} \sum_{q=1}^{p} v_{y, p ; 2 q, q} T_{2 q}{ }_{q+1}^{q} \rho^{p}, \\
& v_{z}=\sum_{p=1}^{\infty} \sum_{q=1}^{p} v_{z, p ; 2 q, q} T_{2 q}{ }^{m}{ }_{q-1} \rho^{p},
\end{aligned}
$$

with

$$
v_{y, p ; 2 q, m}=-\bar{v}_{z, p ; 2 q, m} .
$$

The substitution of this Ansatz in the spinorial counterpart of the conformal Killing operator $(\mathscr{L} v)_{A B C D}$, and then in turn in

$$
D^{A B}\left(\psi_{A B C D}^{\prime}+(\mathscr{L} v)_{A B C D}\right)=0
$$

renders a series of algebraic equations for the coefficients $v_{x, p ; 2 q, q}, v_{y, p ; 2 q, q}$ and $v_{z, p ; 2 q, q}$. Calculations using Maple $\mathrm{V}$ reveal that at least for $p=1, \ldots, 4$, the coefficients $v_{x, p ; 2 p, p}, v_{y, p ; 2 p, p}$ and $v_{z, p ; 2 p, p}$-i.e. for the sector $q=p$ - can be written in terms of the freely specifiable data - i.e. the coefficients $Y_{p ; 2 q, q}^{+}, Z_{p ; 2 q, q}^{-}, L_{0, p ; 2 q, q}, L_{4, p ; 2 q, q}$, and the constants $A, J, Q_{3}$. Now, for $q<p$ the situation is different. It is found that for arbitrary specifiable data, there is in general, no solution to the algebraic equations linking the coefficients $v_{x, p ; 2 q, q}, v_{y, p ; 2 q, q}$ and $v_{z, p ; 2 q, q}$. Nevertheless, conditions for the existence of solutions can be found. These restrictions on the specifiable data can be understood by recalling that generic solutions of the momentum constraint, equation (9b), will have a much more complicated asymptotic behaviour than the one we have imposed through the Ansätze (67a)example. Consequently, conditions on the free data are needed in order to have consistency with the asymptotic behaviour which is being pursued. The conditions are given in the following

Theorem 3. Under assumptions 1, 2 and 3, necessary conditions for the existence of solutions to the momentum constraint, equation (9b), which on $\mathcal{C}_{a}$ are expandable in powers of $\rho$ up to order $\mathcal{O}\left(\rho^{4}\right)$ are

$$
Z_{p ; 2 q, q}^{-}=(-1)^{p+q+1} Y_{p ; 2 q, q}^{+}, \quad p=2, \ldots, 5, \quad q=2, \ldots, p, \quad m=0, \ldots, 2 q,
$$

A further peculiarity of solutions to equation (5.1) under the Ansätze (67a)-(67c) is that even if the conditions given in theorem [3] are satisfied, the coefficients $v_{x, p ; 2 p, q}, v_{y, p ; 2 p, q}$ and $v_{z, p ; 2 p, q}$ are not fully determined by the algebraic conditions implied by the substitution of (67a)-(67c) in equation (5.1). More precisely, if the conditions given in theorem 3 hold then it follows that

$$
v_{y, p ; 2 q, q}+(-1)^{p+q+1} v_{z, p ; 2 q, q}=C_{p ; 2 q, q}
$$

for $p=1, \ldots, 4, q=1, \ldots, p$ and where $C_{p ; 2 q, q}$ denotes some expression depending on the freely specifiable data. Recall that besides, the reality condition (68) must also be satisfied. The reason for this behaviour can be understood as follows. It is well known that the idea behind the York splitting, equation (62), is to obtain an elliptic equation for the 1-form $v_{\alpha}$ out of the underdetermined system (9b). Now, solutions to a system of elliptic equations contain global information about the underlying initial manifold $\mathcal{S}$. This information is not captured if one attempts to calculate formal solutions in a neighbourhood $\mathcal{B}_{a}(i)$ of infinity. This same feature pervades the approach for solving the momentum constraint followed in the previous section. Moreover, a similar phenomenon can be observed when "solving" the Hamiltonian constraint by the same methods — see in particular the discussion in part I. 


\section{Expansions of the Hamiltonian constraint}

As discussed in part I, the Licnerowicz equation, being a scalar equation is easily translated into space spinor language. Indeed, one has

$$
\left(D^{A B} D_{A B}-\frac{1}{8} r\right) \vartheta=\frac{1}{8} \psi_{A B C D} \psi^{A B C D} \vartheta^{-7} .
$$

In order to construct formal expansions of the solutions of this equations on $\mathcal{B}_{a}(i)$-respectively $\mathcal{C}_{a}$ - we adopt the local parametrisation

$$
\vartheta=\frac{U}{|x|}+W
$$

The term $U /|x|$ (the Green function) contains information regarding the local geometry near infinity. In the conformally flat case on has $U=1$. On the other hand, the term $W$ contains information of a global nature. For example, $W(i)=m / 2$, where $m$ is the ADM mass of the initial data set $\left(\widetilde{\mathcal{S}}, \widetilde{h}_{\alpha \beta}, \widetilde{\chi}_{\alpha \beta}\right)$ - the global quantity par excellence.

\subsection{The construction of the Green function}

In order to calculate the function $U$, we shall make use of Hadamard's parametrix construction -see e.g. [8, 10]. The term $U /|x|$, being the Green's function of the Yamabe operator - see below-, corresponds to a solution of the equation

$$
\left(D^{\alpha} D_{\alpha}-\frac{1}{8} r\right)\left(\frac{U}{|x|}\right)=4 \pi \delta_{i},
$$

where $\delta_{i}$ is the Dirac distribution centred in $i$. Now, consider a function $U$ of the form

$$
U=\sum_{p=0}^{\infty} U_{p}|x|^{2 p},
$$

where $U_{p}=U_{p}(|x|)$, that is, the coefficients in the Ansatz are still allowed to have $\rho$-dependence. Under this Ansatz, equation (74) implies the following hierarchy of ordinary differential equations:

$$
\begin{aligned}
& D^{\alpha} \rho^{2} D_{\alpha} U_{0}=-\frac{1}{2}\left(D^{\alpha} D_{\alpha} \rho^{2}+6\right) U_{0}, \quad U(i)=1, \\
& D^{\alpha} \rho^{2} D_{\alpha} U_{p}=-\frac{1}{2}\left(D^{\alpha} D_{\alpha} \rho^{2}+6-4 p\right) U_{p}-\frac{1}{2 p-1} L_{h}\left[U_{p-1}\right], \quad p=1,2, \ldots
\end{aligned}
$$

where

$$
L_{h}[f]=\left(D^{\alpha} D_{\alpha}-1 / 8 r\right) f
$$

denotes the Yamabe operator of the metric $h_{\alpha \beta}$ applied to a smooth function $f$. The solutions to the above equations can be given recursively by

$$
\begin{aligned}
& U_{0}=\exp \left(\frac{1}{4} \int_{0}^{\rho}\left(D^{\alpha} D_{\alpha} s^{2}+6\right) \frac{\mathrm{d} s}{s}\right), \\
& U_{p+1}=-\frac{U_{0}}{(4 p-2) \rho^{p+1}} \int_{0}^{\rho} \frac{L_{h}\left[U_{p}\right] s^{p}}{U_{0}} \mathrm{~d} s, \quad p=0,1, \ldots
\end{aligned}
$$

Now, given a conformal 3-metric $h_{\alpha \beta}$, the above procedure does not necessarily render a convergent $U$ as given by the series (75). One needs extra assumptions on $h$. For example, if $h_{\alpha \beta}$ is analytic in a neighbourhood of $i$ then $U$ given by (75) is also analytic.

Under the more general requirements of assumption 2 the best one can hope for is a function $U$ of a similar form -i.e. $U=U^{(1)}+\rho U^{(2)}$. The latter will be the case only if further conditions on the conformal metric hold, for some experimentation reveals that free data of the form given by assumption 2 render coefficients $U_{p}$ with logarithmic terms in $|x|$. Direct calculations with Maple $\mathrm{V}$ yield the following 
Theorem 4. Under assumption 圆 the coefficients $U_{0}, \ldots, U_{5}$ have a logarithm-free dependence on $|x|$ if and only if

$$
\begin{aligned}
& Z_{2 ; 4,2}^{-}=-Y_{2 ; 4,2}^{+}, \\
& Y_{3 ; 0,0}^{-}=0, \quad Y_{3 ; 2,1}^{-}=0 \\
& Z_{3 ; 6,3}^{-}=-Y_{3 ; 6,3}^{+}, \\
& Z_{4 ; 4,2}^{-}=-Y_{4 ; 4,2}^{+}, \quad Z_{4 ; 8,4}^{-}=-Y_{4 ; 8,4}^{+}, \\
& Z_{5 ; 6,3}^{-}=-Y_{5 ; 6,3}^{+}, \quad Z_{5 ; 10,5}^{-}=-Y_{5 ; 10,5}^{+} .
\end{aligned}
$$

If the above conditions hold, then

$$
\begin{aligned}
U_{0} & =1+\mathcal{O}\left(|x|^{4}\right), \\
U_{1} & =\mathcal{O}\left(|x|^{2}\right), \\
U_{2} & =\mathcal{O}\left(|x|^{2}\right), \\
U_{3} & =\mathcal{O}\left(|x|^{0}\right), \\
U_{4} & =\mathcal{O}\left(|x|^{-1}\right), \\
U_{5} & =\mathcal{O}\left(|x|^{-3}\right) .
\end{aligned}
$$

Remark. The conditions given in theorem 4 allow to calculate an expansion for $U$ up to order $\mathcal{O}\left(\rho^{7}\right)$.

\subsection{Construction of expansions of $W$}

The substitution of the parametrisation (73) in the Hamiltonian constraint, equation (9a), renders the following equation for the function $W$ in $\mathcal{C}_{a}$ :

$$
\left(D^{A B} D_{A B}-\frac{1}{8} r\right) W=\frac{1}{8} \psi_{A B C D} \psi^{A B C D}(U / \rho+W)^{-7} .
$$

Under the assumption of axial symmetry (assumption 0), we shall be looking for solutions on $\mathcal{C}_{a}$ of the form

$$
W=m / 2+\sum_{p=1}^{\infty} \sum_{q=0}^{p} \frac{1}{p !} w_{p ; 2 q, q} T_{2 q}{ }_{q}^{q} \rho^{p},
$$

with $w_{p ; 2 q, m} \in \mathbb{R}$ so that $W$ is a real function on $\mathcal{C}_{a}$.

As in the conformally flat case discussed in part I, the substitution of the Ansatz (79) into equations (78) allows to determine the coefficients $w_{p ; 2 q, q}$, with $q<p$. The coefficients $w_{p ; 2 p, p}$ - like the ADM mass itself - are, on the other hand, not fixed by this procedure, and shall remain free in our construction. Note, however, that in an actual solution of the constraints, these coefficients are functions of the free data. This dependence can be analysed, for example, by writing an integral representation of the solution - see e.g. the discussion in part I or in [5].

In part I, it was shown that the dipolar terms in the expansions of $W$ can be, without loss of generality, chosen to be zero. This could be done because our setup still allowed for a translational freedom. In our case, due to the use of cn-gauge to fix the conformal gauge this is in general not possible. Nevertheless, in order to ease the complexity of our computations we make the following

Assumption 4. We shall restrict our assumption to initial data sets such that the function $W$ in the parametrisation $\vartheta=U / \rho+W$ of the conformal factor is of the form

$$
W=\frac{m}{2}+\mathcal{O}\left(|x|^{2}\right)
$$

Assumption 4 implies that in the expansion (79) one has $w_{1 ; 2, m}=0$ for $m=0,1,2$. For the purposes of this article the expansions of $W$ have been explicitly calculated up to order $\mathcal{O}\left(\rho^{7}\right)$. 


\section{Regularity conditions on the initial data}

As in part I, let us expand the components of the Weyl spinor $\phi_{A B C D}$ as

$$
\phi_{j}=\sum_{p=|2-j|}^{\infty} \frac{1}{p !} \sum_{q=|2-j|}^{p} \sum_{m=0}^{2 q} a_{j, p ; 2 q, m} T_{2 q}{ }^{m}{ }_{q-2+j} \rho^{p},
$$

where the (complex) coefficients $a_{j, p ; 2 q, m}$ have $\tau$ dependence - that is, $a_{j, p ; 2 q, m}=a_{j, p ; 2 q, m}(\tau)$. Friedrich - see [8] - has shown that the solutions to the transport equations implied by the conformal Einstein equations at spatial infinity develop a certain type of logarithmic singularity at the sets where null infinity "touches" spatial infinity unless the initial data is such that

$$
a_{0, p ; 2 p, k}(0)=a_{4, p ; 2 p, k}(0)
$$

with $p=2,3, \ldots$, and $k=0, \ldots, 2 q$ holds.

In part I, the regularity condition (80) was rephrased in terms of the freely specifiable terms available in the case of conformally flat initial data sets. It essentially implied that the coefficients $L_{0, p-4 ; 2 p, k}$ and $L_{4, p-4 ; 2 p, k}$ encoding part of the higher multipole structure of the conformally flat initial data sets have to be alternatively real or pure imaginary numbers depending on the parity of $k$-see theorem 3 in [17.

In the case of initial data of the form being considered here, we have the following

Theorem 5. For an initial data set satisfying the assumptions 1-4, the following conditions are equivalent:

(i) $\phi_{0, p ; 2 p, p}(0)=\phi_{4, p ; 2 p, p}(0), p=2,3,4,5,6$;

(ii)

$$
\begin{aligned}
Y_{2 ; 4,2}^{+} & =\frac{2}{3}\left(L_{4,-2 ; 4,2}+L_{0,-2 ; 4,2}\right), \\
Y_{3 ; 6,3}^{+} & =\frac{9}{4}\left(L_{4,-1 ; 6,3}+L_{0,-1 ; 6,3}\right), \\
Y_{4 ; 8,4}^{+} & =\frac{96}{5}\left(L_{0,0 ; 8,4}+L_{4,0 ; 8,4}\right), \\
Y_{5 ; 10,5}^{+} & =-100\left(L_{0,1 ; 10,5}+L_{4,1 ; 10,5}\right), \\
Y_{6 ; 12,6}^{+} & =-\frac{1080}{7}\left(L_{0,2 ; 12,6}+L_{4,2 ; 12,6}\right) .
\end{aligned}
$$

This result naturally leads to our final assumption.

Assumption 5. The second fundamental form satisfies the condition (ii) in theorem 5 .

\section{Solutions to the transport equations and obstructions to the smoothness of null infinity}

We shall now proceed, in a parallel way as it was done in part I, to discuss the properties of the solutions of the transport equations implied by the conformal Einstein equations - see equations (131), (132) and (133) - in the case of initial data sets satisfying assumptions 0-5. For details on the structure of the transport equations we remit the reader to part I. We just recall that the transport equations implied by the conformal Einstein equations upon evaluation on the cylinder at spatial infinity naturally group in two subsystems. One, called the $v$ subsystem, contains the evolution equations for the frame, connection, and the components of the Ricci tensor of the Weyl connection. The other one, the so-called Bianchi subsystem, provides equations for the components of the Weyl tensor. As in references 15, 14, 17, we shall be restricting our attention to the logarithmic terms arising in the components of the Weyl spinor $\phi_{A B C D}$ - the reasons for 
this way of proceeding have been discussed in the aforementioned references. To this end we recall that we are decomposing the components of the Weyl tensor as

$$
\phi_{j}=\sum_{p=|2-j|}^{\infty} \frac{1}{p !} \phi_{j}^{(p)} \rho^{p},
$$

where

$$
\phi_{j}^{(p)}=\sum_{q=|2-j|}^{p} \sum_{k=0}^{2 q} a_{j, p ; 2 q, k}(\tau) T_{2 q}{ }^{k} q-2+j .
$$

Retaking a notation already used in part I, we use $\mathcal{Q}(\tau)$ to denote a generic polynomial in $\tau$, while $\mathcal{P}_{k}(\tau)$ will denote a generic polynomial of degree $k$ in $\tau$ such that $\mathcal{P}_{k}( \pm 1)=0$. It will be understood that the $\mathcal{Q}(\tau)$ and $\mathcal{P}_{k}(\tau)$ appearing in different equations and/or components are also different from each other.

\subsection{Lower order solutions}

Our first result is the following

Theorem 6. Under assumptions 0-5, the solutions of both the $v$ and Bianchi transport equations for $p=0,1,2$ are polynomial in $\tau$. In addition, the solutions of the $v$ transport equations are polynomial for $p=3$.

A first difference with respect to the cases analysed in paper I and also in 15, 14, 11, is that extra conditions are needed to calculate the solutions of the $p=3$ Bianchi transport subsystem. Namely, one has that

Theorem 7. Under assumptions 1-5, the Bianchi transport constraint equations are satisfied if and only if

$$
L_{4,-2 ; 4,2}=-L_{0,-2 ; 4,2} .
$$

Furthermore, if the latter condition holds then the solutions of the Bianchi transport equations for $p=3$ are polynomial in $\tau$.

Remark. It is noted that the condition 844) arose in paper I as a regularity condition which precluded the appearance of logarithms associated to the "highest possible" harmonics. In the present case 84) implies, in turn, that

$$
Y_{2 ; 4,2}^{+}=Y_{2 ; 4,2}^{-}=Z_{2 ; 4,2}^{+}=Z_{2 ; 4,2}^{-}=0,
$$

from where it follows that $\check{c}_{A B}^{ \pm}=\mathcal{O}\left(\rho^{3}\right)$.

\subsection{The solutions at order $p=4$}

Now, let the assumptions 1-5 together with the condition (84) hold. The CA computations show that the solutions to the $p=4 \mathrm{v}$ transport equations are, as it is to be expected, polynomial in $\tau$. In complete analogy to the $p=3$ case one has that some extra conditions need to be satisfied in order to be able to solve the $p=4$ Bianchi transport equations. More precisely, one has the following

Theorem 8. Let assumptions 0-5 together with condition 84). Then the $p=4$ Bianchi constraint equations are satisfied if and only if

$$
L_{4,-1 ; 6,3}=-L_{0,-1 ; 6,3} .
$$

Furthermore the functions $a_{j, 4 ; 2 q, q}(\tau), q=j, \ldots, 4 q \neq 2$ are polynomial in $\tau$, while

$$
a_{j, 4 ; 4,2}(\tau)=\Upsilon_{4 ; 4,2}\left((1-\tau)^{6-j} \ln (1-\tau) \mathcal{P}_{j}(\tau)+(1+\tau)^{2+j} \ln (1+\tau) \mathcal{P}_{4-j}(\tau)\right)+\mathcal{Q}(\tau),
$$

where

$$
\Upsilon_{4 ; 4,2}=Z_{3 ; 4,2}^{-}-Y_{3 ; 4,2}^{+} \text {. }
$$


Remark 1. Again, because of the regularity conditions given in theorem [5] if the condition (85) holds then one has that

$$
Y_{3 ; 6,3}^{+}=Y_{3 ; 6,3}^{-}=Z_{3 ; 6,3}^{+}=Z_{3 ; 6,3}^{-}=0 .
$$

Remark 2. The obstruction (87) is a result of the non-conformal flatness of our setting. It did not arise in the analysis carried out in 14] as the free data was chosen in such a way that it was automatically satisfied. It is noted that had we solved the momentum constraint in the way indicated in section 5.1 then the obstruction (87) would not have arosen - see theorem 3 . As it will be seen in the sequel, analogous higher order obstruction would have, similarly, gone unnoticed.

\subsubsection{Solutions at order $p=5$}

For the solutions of the $p=5$ transport equations one has the following

Theorem 9. Consider an initial data set satisfying the conditions 0-5. Assume, additionally, that the conditions (84), (85) also hold and the obstruction (87) vanishes. Then the solutions to the $p=5 v$ transport equations are polynomial in $\tau$ and the transport equations implied by the Bianchi constraint equations at order $p=5$ are satisfied if and only if

$$
L_{4,0 ; 8,4}=-L_{0,0 ; 8,4} .
$$

If the latter holds one has that $a_{j, 5 ; 2 q, q}(\tau), j=0, \ldots, 4, q=|2-j|, \ldots, 5$ with $q \neq 2,3$ are polynomial in $\tau$ and

$$
a_{j, 5 ; 4,2}(\tau)=\Upsilon_{5 ; 4,2}\left((1-\tau)^{7-j} \mathcal{P}_{j}(\tau) \ln (1-\tau)+(1+\tau)^{3+j} \mathcal{P}_{4-j}(\tau) \ln (1+\tau)\right)+\mathcal{Q}(\tau),
$$

where

$$
\begin{aligned}
\Upsilon_{5 ; 4,2}= & 18 w_{2 ; 4,2} m^{2}+m R_{2 ; 4,2}+\frac{37602}{199} m J^{2}-\frac{3099}{199} \sqrt{6} m^{2} L_{0,-2 ; 4,2} \\
& +\frac{1023}{199} \sqrt{6} m\left(L_{0,-1 ; 4,2}-L_{4,-1 ; 4,2}\right)+\frac{224}{199} \sqrt{6}\left(L_{0,0 ; 4,2}-L_{4,0 ; 4,2}\right) .
\end{aligned}
$$

Furthermore,

$$
a_{j, 5 ; 6,3}(\tau)=\Upsilon_{5 ; 6,3}\left((1-\tau)^{7-j} \ln (1-\tau) \mathcal{P}_{j+1}+(1+\tau)^{3+j} \ln (1+\tau) \mathcal{P}_{5-j}(\tau)\right)+\mathcal{Q}(\tau),
$$

where

$$
\Upsilon_{5 ; 6,3}=Z_{4 ; 6,3}^{-}-Y^{+} 2_{4 ; 6,3} .
$$

Remark 1. The expression (91) is the natural generalisation of the quadrupolar obstructions obtained in paper I and in [15, 14]. In particular, in order to make the comparison with the results of [14] more transparent we note that the calculations leading to lemma 4 in section 3.2 show that

$$
r_{2 ; 4,2}=16 \sqrt{6} Y_{3 ; 4,2}^{+},
$$

where

$$
r=r_{2 ; 4,2} T_{4}{ }_{2}^{2} \rho^{2}+\mathcal{O}\left(\rho^{3}\right),
$$

and $r$ is the Ricci scalar of the conformal metric as discussed in section 3. Thus, in the time symmetric case -i.e. if $J=L_{0,-2 ; 4,2}=L_{0,-1 ; 4,2}=L_{4,-1 ; 4,2}=L_{0,0 ; 4,2}=L_{4,0 ; 4,2}=0$ - the quadrupolar obstruction (91) reduces to

$$
\Upsilon_{5 ; 4,2}=18 w_{2 ; 4,2} m^{2}+m R_{2 ; 4,2} .
$$

Remark 2. Note that the first three terms in (91) are manifestly real, while the remaining three are - due to the reality conditions and the condition (84) in theorem 7-pure imaginary. Thus, the vanishing of the quantity $\Upsilon_{5 ; 4,2}$ implies, on the one hand, that

$$
m w_{2 ; 4,2}=-\frac{8 \sqrt{6}}{9} Y_{3 ; 4,2}^{+}-\frac{2089}{199} J^{2},
$$


and on the other hand

$$
1023 m\left(L_{0,-1 ; 4,2}-L_{4,-1 ; 4,2}\right)+224\left(L_{0,0 ; 4,2}-L_{4,0 ; 4,2}\right)-3099 m^{2} L_{0,-2 ; 4,2}=0 .
$$

Remark 3. As in the case of the quadrupolar obstruction (87), the octupolar obstruction (93) is purely due to the non-conformal flatness of the set up and did not arise in the analysis given in 14] as the initial data considered in that case was constructed so as to satisfy it automatically.

\subsection{Solutions at order $p=6$}

The expansions at order $p=6$ are both quantitatively and qualitatively more complex than those of the previous orders. Firstly, we discuss which conditions are needed to ensure the existence of solutions. These are given in the next

Lemma 6. Consider initial data satisfying the assumptions 0-5, and such that that the conditions 84), (85) and (89) hold. Furthermore, assume that the initial data is such that the obstructions 87), (91) and (93) vanish. Then the solutions to the $p=6 v$ transport equations are polynomial in $\tau$ and the $p=6$ transport equations implied by the Bianchi constraint equations are satisfied if and only if

$$
L_{0,1 ; 10,5}=-L_{4,1 ; 10,5}
$$

The structure of the solutions to the $p=6$ Bianchi transport equations is described in the following theorem.

Theorem 10. Consider initial data sets satisfying the assumptions 0-5, and such that the conditions 84), 851), 89) and (98) hold. Furthermore, assume that the initial data is such that the obstructions 87), 91) and (93) vanish. Then the coefficients $a_{j, 6 ; 2 q, q}(\tau)$, with $j=0, \ldots, 4$, $q=|2-j|, \ldots, 6, q \neq 2, q \neq 3$ have polynomial dependence in $\tau$. On the other hand,

$$
a_{j, 6 ; 4,2}=\Upsilon_{6 ; 4 ; k}^{+}(1-\tau)^{8-j} \mathcal{P}_{j}(\tau) \ln (1-\tau)+\Upsilon_{6 ; 4,2}^{-}(1+\tau)^{4+j} \mathcal{P}_{4-j}(\tau) \ln (1+\tau)+\mathcal{Q}(\tau),
$$

where the obstructions are given by

$$
\begin{aligned}
\Upsilon_{6 ; 4,2}^{+}= & \frac{722}{7} i m J Q_{3}-\frac{2198208}{6965} m^{2} J^{2}-\frac{20817}{14} A m J^{2} \\
& +\frac{62691}{4816} \sqrt{6} A m\left(L_{4,-1 ; 4,2}-L_{0,-1 ; 4,2}\right)-\sqrt{6} m^{2}\left(\frac{7559711}{126420} L_{4,-1 ; 4,2}+\frac{8613019}{126420} L_{0,-1 ; 4,2}\right) \\
& +\frac{58}{43} \sqrt{6} A\left(L_{4,0 ; 4,2}-L_{0,0 ; 4,2}\right)+\sqrt{6} m\left(\frac{3282401}{27090} L_{4,0 ; 4,2}+\frac{3648769}{27090} L_{0,0 ; 4,2}\right) \\
& +\frac{144}{13} \sqrt{6}\left(L_{4,1 ; 4,2}+L_{0,1 ; 4,2}\right) \\
& -\frac{11500}{91} \sqrt{6} A m Y_{3 ; 4,2}^{+}-\frac{2985}{56} \sqrt{6} m Y_{4 ; 4,2}^{+}+\frac{18}{35} \sqrt{6}\left(Z_{5 ; 4,2}^{-}-Y_{5 ; 4,2}^{+}\right), \\
\Upsilon_{6 ; 4,2}^{-}= & 722 \frac{2 m J Q_{3}}{7}+\frac{2198208}{6965} m^{2} J^{2}-\frac{20817}{14} A m J^{2} \\
& +\frac{62691}{4816} \sqrt{6} A m\left(L_{4,-1 ; 4,2}-L_{0,-1 ; 4,2}\right)-\sqrt{6} m^{2}\left(\frac{7559711}{126420} L_{0,-1 ; 4,2}+\frac{8613019}{126420} L_{4,-1 ; 4,2}\right) \\
& +\frac{58}{43} \sqrt{6} A\left(L_{4,0 ; 4,2}-L_{0,0 ; 4,2}\right)+\sqrt{6} m\left(\frac{3282401}{27090} L_{0,0 ; 4,2}+\frac{3648769}{27090} L_{4,0 ; 4,2}\right) \\
& +\frac{144}{13} \sqrt{6}\left(L_{4,1 ; 4,2}+L_{0,1 ; 4,2}\right) \\
& -\frac{11500}{91} \sqrt{6} A m Y_{3 ; 4,2}^{+}-\frac{2985}{56} \sqrt{6} m Y_{4 ; 4,2}^{+}+\frac{18}{35} \sqrt{6}\left(Z_{5 ; 4,2}^{-}-Y_{5 ; 4,2}^{+}\right) .
\end{aligned}
$$

Moreover,

$$
a_{j, 6 ; 6,3}(\tau)=\Upsilon_{6 ; 6,3}\left((1-\tau)^{8-j} \mathcal{P}_{j+1}(\tau) \ln (1-\tau)+(1+\tau)^{4+j} \mathcal{P}_{5-j}(\tau) \ln (1+\tau)\right)+\mathcal{Q}(\tau),
$$


where

$$
\begin{aligned}
\Upsilon_{6 ; 6,3}= & 24 m^{3} w_{3,6,3}-\frac{565753248}{82585} i m^{2} J^{3} \\
& +\frac{36399}{830} \sqrt{6} i m^{2} J\left(L_{4,-1,4,2}-L_{0,-1 ; 4,2}\right)+\frac{1904}{415} \sqrt{6} i m J\left(L_{4,0 ; 4,2}-L_{0,0 ; 4,2}\right) \\
& -\frac{7272}{415} \sqrt{30} m L_{0,-1,6,3}+\frac{408}{83} \sqrt{30} m^{2}\left(L_{0,0 ; 6,3}-L_{4,0 ; 6,3}\right)+\frac{576}{415} \sqrt{30}\left(L_{0,1 ; 6,3}-L_{4,1 ; 6,3}\right) \\
& -\frac{233216}{415} \sqrt{6} i m J Y_{3 ; 4,2}^{+}+6 \sqrt{30} Y_{4 ; 6,3}^{+} .
\end{aligned}
$$

Remark 1. The most noticeable feature of the order $p=6$ quadrupolar obstructions (100a) and $100 \mathrm{~b}$ is that they are time asymmetric. That is, the vanishing of one of them does not imply the vanishing of the other at least if $J \neq 0$-this feature had already appeared in the conformally flat case discussed in paper I. However, it is noted that at least to this order the conformal flatness plays no role in the time asymmetric behaviour.

Remark 2. If both $\Upsilon_{6 ; 4,2}^{+}$and $\Upsilon_{6 ; 4,2}^{-}$vanish then the expressions 100a and 100b can be used to solve for, say, $L_{0,-1 ; 4,2}$ and $L_{4,-1 ; 4,2}$ in a similar way to how it was done in paper I.

\section{Conjecture about the general structure of the solutions}

The results of the CA calculations carried out in the previous section exhibit a well defined structure. From here and together with the results of part I, it is not hard to conjecture what the behaviour of the solutions for a generic order $p$ should be.

Firstly, we note that the conditions (84), (85), (89) and (98) arise, arguably, as a side effect of the way we have calculated expansions consistent with the momentum constraint (9b) and that strictly speaking we have not found solutions. It is quite likely that the use of explicit solutions to the momentum constraint — as it is possible in the axially symmetric case, see section 2 - would get rid of such conditions. Therefore, conditions of this sort will be disregarded in the subsequent discussion. Moreover, following the general trend of the paper, we limit ourselves to the axially symmetric case. The generalisation of the discussion given in the sequel for non-axially symmetric initial data sets should be direct.

As with the expansions carried out in section 5, we restrict ourselves to a class of initial data sets satisfying assumptions 0-5. Now, assuming that for a given integer $p_{*} \geq 7$ all lower order obstructions vanish -i.e. those arising from the $p=4, \ldots, p_{*}-1$ Bianchi constraint equationsit is conjectured that the solutions to the Bianchi transport equations are such that:

(i) The coefficients

$$
a_{j, p ; 2 p, p}(\tau), \quad a_{j, p ; 2(p-1), p-1}(\tau), \quad j=0, \ldots, 4,
$$

associated with the harmonics $T_{2 p}^{p}{ }_{p+j}$ and $T_{2(p-1)}^{p-1}{ }_{p-1+j}$, respectively, are polynomials in $\tau$.

(ii) The coefficients $a_{j, p ; 2(p-2), p-2}$ accompanying the harmonics $T_{2(p-2)}^{p-2}{ }_{p-2+j}$ are of the form

$$
\begin{aligned}
a_{j, p ; 2(p-2), p-2}(\tau)=\Upsilon_{p ; 2(p-2), p-2}\left((1-\tau)^{p+2-j}\right. & \ln (1-\tau) \mathcal{P}_{p-4+j}(\tau) \\
& \left.+(1+\tau)^{p-2-j} \ln (1+\tau) \mathcal{P}_{p-j}(\tau)\right)+\mathcal{Q}(\tau)
\end{aligned}
$$

where the obstruction $\Upsilon_{p ; 2(p-2), p-2}$ is given by

$$
\Upsilon_{p ; 2(p-2), p-2}=Z_{p-1 ; 2(p-2), p-2}^{-}-Y_{p-1 ; 2(p-2), p-2}^{+} .
$$


(iii) The coefficients $a_{j, p ; 2(p-3), p-3}$ which go together with the harmonics $T_{2(p-3)}^{p-3}{ }_{p-3+j}$ are of the form

$$
\begin{aligned}
a_{j, p ; 2(p-3), p-3}(\tau)=\Upsilon_{p ; 2(p-3), p-3}\left((1-\tau)^{p+2-j}\right. & \ln (1-\tau) \mathcal{P}_{p-5+j}(\tau) \\
& \left.+(1+\tau)^{p-2+j} \ln (1+\tau) \mathcal{P}_{p-1-j}(\tau)\right)+\mathcal{Q}(\tau)
\end{aligned}
$$

where $\Upsilon_{p ; 2(p-3), p-3}$ is a time symmetric obstruction similar in structure to those given by (91) and (102), and should be a quantity of $2^{p-3}$-polar nature. That is, it should be possible to write it as a linear combination of quantities quadrupolar nature like

$$
w_{p-3 ; 2(p-3), p-3}, \quad Y_{p-2 ; 2(p-3), p-3}^{+}, \quad\left(L_{4, s ; 2(p-3), p-3}-L_{0, s ; 2(p-3), p-3}\right),
$$

and $2^{p-3}$-polar products of lower multipole quantities such as

$$
J^{p-3}, \quad J Y_{p-3 ; 2(p-4), p-4}^{+}, \quad J\left(L_{4, s ; 2(p-4), p-4}-L_{0, s ; 2(p-4), p-4}\right) .
$$

(iv) The coefficients $a_{j, p, 2 q, q}$ for $q=2, \ldots, p-4$ have also logarithmic dependence, being of the form

$$
\begin{aligned}
a_{j, p ; 2 q, q}(\tau)=\Upsilon_{p ; 2 q, q}^{+}(1-\tau)^{p+2-j} \ln (1-\tau) \mathcal{P}_{q+j-2}(\tau) & \\
& +\Upsilon_{p ; 2 q, q}^{-}(1+\tau)^{p-2+j} \mathcal{P}_{q+2-j}(\tau)
\end{aligned}
$$

where $\Upsilon_{p ; 2 q, q}^{+}$and $\Upsilon_{p ; 2 q, q}^{-}$are obstructions of $2^{q}$-polar nature. Furthermore,

$$
\Upsilon_{p ; 2 q, q}^{+}=0 \nLeftarrow \Upsilon_{p ; 2 q, q}^{-}=0,
$$

that is, the obstructions are time asymmetric.

(v) The coefficients associated with harmonics of the form $T_{2}{ }_{1}^{1}$ and $T_{0}{ }_{0}^{0}$, that is $a_{j, p ; 2,1}(\tau)$, $j=1,2,3$ and $a_{2, p ; 0,0}(\tau)$ are polynomials in $\tau$.

Providing a proof of the above structure would constitute a remarkable feat. As things stand now, it would require a much deeper understanding of the group theoretical structures underlying the cylinder at spatial infinity, $\mathcal{I}$.

\section{Conclusions}

The discussion presented in the current article is the natural extension of the analyses carried out in [15, 14, 17. The objective at the start of this programme was to provide evidence for a conjecture presented by H. Friedrich in [9]. As discussed at length elsewhere, this condition stated that only mild asymptotic conditions on the initial data were required in order to guarantee the existence of developments with a smooth null infinity. The conjectured asymptotic requirements were essentially that condition (i) in 5 holds to all orders. As shown in [15 this conjecture resulted too optimistic. The results in [15, 14, 17] have suggested that Penrose's notion of asymptotic simplicity imposes stringent restrictions on the behaviour of the gravitational field in the socalled region of spacetime "close to null and spatial infinity". Namely, that the field should be static/stationary in this region - a startling rigidity result if proved to be true.

The basis of this new conjecture was the discovery of a hierarchy of so-called obstructions to the smoothness of null infinity. Roughly, these obstructions are quantities which can be fully determined in terms of initial data sets and whose vanishing eliminates some particular singular logarithmic behaviour at the sets where null infinity "touches" spatial infinity. Some of these obstructions can be fully written in terms of the freely specifiable data - like the ones appearing in section (ii) and (iv) of section 9- while others involve the coefficients $w_{p ; 2 q, q}$ which arise 
from the expansions of the conformal factor of the initial hypersurface $\vartheta$. The latter class of obstructions is much more complex, as it is not clear - at least at first sight - how can one construct data for which these obstructions vanish; the reason being that the coefficients $w_{p ; 2 q, q}$ are not freely specifiable. They are part of the solution to an elliptic equation (at least if one uses the conformal method to solve the constraints), and contain information of global nature. They are, in principle, (complicated) functions of the free data.

In paper I, it was shown that the assumption regarding obstructions up to a certain order $(p=8)$ vanish leads naturally to initial data sets which are asymptotically Schwarzschildean. Ideally, in the present paper, one would like to repeat the same type of procedure with the obstructions we have been calculated, however they seem to be insufficient to be able to extract some type of statement concerning asymptotic stationarity. The way stationary solutions fit in the whole scheme, will be the subject of further study.

Finally, we would like to make a remark concerning a point that has been implicit through the whole programme carried out in [15, 14, 17] and being concluded in the present article. Namely, that the hierarchy of obstructions to the smoothness we have been discussed are also obstructions to the peeling behaviour of the spacetimes, at least in what it concerns the lower order obstructions: $\Upsilon_{4 ; 4 ; 2}, \Upsilon_{5 ; 4,2}, \Upsilon_{5 ; 6,3}$. Moreover, there are reasons to believe that the constancy of the NewmanPenrose constants would involve the obstructions arising at order $p=6$-i.e. $\Upsilon_{6 ; 4,2}^{ \pm}, \Upsilon_{6 ; 6,3}$ and $\Upsilon_{6 ; 8,4}$. The first steps to disentangle this connection have been sketched in [16. A more complete discussion of this connection will be given elsewhere.

\section{Acknowledgments}

I want to thank R. Beig, H. Friedrich for encouragement and helpful discussions. Further gratitude is due to S. Dain for insightful conversation and for sharing unpublished results which give rise to part of the content of section 2 - theorem 1 and2 Gratitude is also due to CM Losert for a careful reading of the manuscript. The computer algebra which constitute the core of the investigation were carried out in the computers of the Max Planck Institute für Gravitationsphysik, Albert Einstein Institute in Golm, Germany. This research is funded by the Austrian FWF (Fonds zur Förderung der Wissenschaftliche Forschung) via a Lise Meitner fellowship (M690-N02 \& M814N02).

\section{References}

[1] R. Beig \& W. Simon, The stationary gravitational field near spatial infinity, Gen. Rel. Grav. 12, 1003 (1980).

[2] S. Dain, Private communication.

[3] S. Dain, Initial data for stationary spacetimes near spacelike infinity, Class. Quantum Grav. 18, 4329 (2001).

[4] S. Dain, Initial data for two Kerr-like black holes, Phys. Rev. Lett. 87, 121102 (2001).

[5] S. Dain, Black hole interaction energy, Phys. Rev. D 66, 084019 (2002).

[6] S. Dain, Asymptotically flat and regular Cauchy data, in The conformal structure of spacetime. Geometry, analysis, numerics, edited by J. Frauendiener \& H. Friedrich, page 161, Springer, 2003.

[7] S. Dain \& H. Friedrich, Asymptotically flat initial data with prescribed regularity at infinity, Comm. Math. Phys. 222, 569 (2001).

[8] H. Friedrich, Gravitational fields near space-like and null infinity, J. Geom. Phys. 24, 83 (1998). 
[9] H. Friedrich, Conformal Einstein evolution, in The conformal structure of spacetime: Geometry, Analysis, Numerics, edited by J. Frauendiener \& H. Friedrich, Lecture Notes in Physics, page 1, Springer, 2002.

[10] H. Friedrich, Smoothness at null infinity and the structure of initial data, in 50 years of the Cauchy problem in general relativity, edited by P. T. Chruściel \& H. Friedrich, Birkhausser, 2004.

[11] H. Friedrich \& J. Kánnár, Bondi-type systems near space-like infinity and the calculation of the NP-constants, J. Math. Phys. 41, 2195 (2000).

[12] V. Moncrief, Spacetime symmetries and linearization stability of the Einstein equations. I., J. Math. Phys. 16, 493 (1975).

[13] J. A. Valiente Kroon, Asymptotic expansions of the Cotton-York tensor on slices of stationary spacetimes, Class. Quantum Grav. 21, 3237 (2004).

[14] J. A. Valiente Kroon, Does asymptotic simplicity allow for radiation near spatial infinity?, Comm. Math. Phys. 251 (2004).

[15] J. A. Valiente Kroon, A new class of obstructions to the smoothness of null infinity, Comm. Math. Phys. 244, 133 (2004).

[16] J. A. Valiente Kroon, On the nonexistence of conformally flat slices for the Kerr and other stationary spacetimes, Phys. Rev. Lett. 92, 041101 (2004).

[17] J. A. Valiente Kroon, Time asymmetric spacetimes near null and spatial infinity. I. Expansions of developments of conformally flat data, Class. Quantum Grav. 23, 5457 (2004). 